$B B$
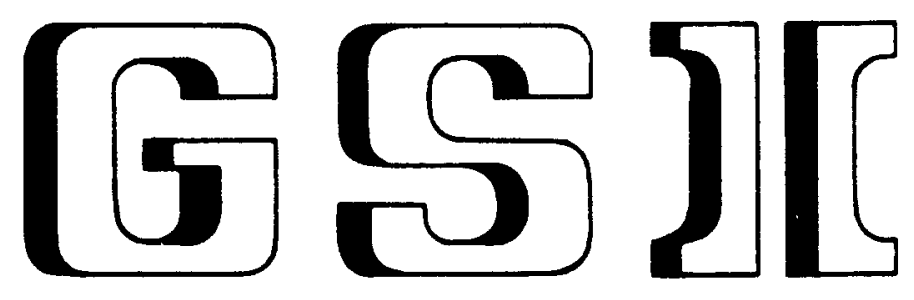

GSI-Preprint-96-01

JANUAR 1996

STUDY OF THE OUT-OF-PLANE EMISSION OF PROTONS

AND LIGHT FRAGMENTS IN SYMMETRIC HEAVY-ION COLLISIONS

D. BRILL, P. BECKERLE, C. BORMANN, E. SCHWAB, Y. SHIN, R. STOCK,

H. STRÖBELE, P. BALTES, C. MÜNTZ, H. OESCHLER, C. STURM, A. WAGNER,

R. BARTH, M. CIESLAK, M. DEBOWSKI, E. GROSSE, P. KOCZON. M. MANG,

D. MISKOWIEC, R. SCHICKER, P. SENGER, B. KOHLMEYER, F. PÜHLHOFER,

J. SPEER, K. VÖLKEL, W. WALUS

(accepted for publ. in Z. Phys. A.)

CERN LIBRARIES, GENEVA

Quality insufficient for good scanning

Gesellschaft für Schwerionenforschung $\mathrm{mbH}$

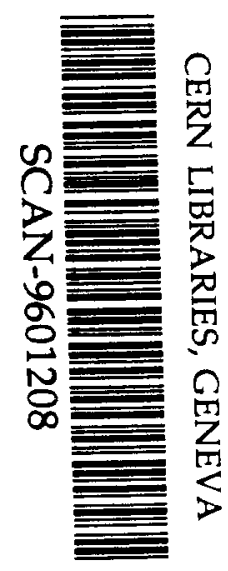

Postfach 110552 D-64220 Darmstadt · Germany

sw9606 


\title{
Study of the Out-of-Plane Emission of Protons and Light Fragments in Symmetric Heavy-Ion Collisions
}

\author{
D. Brill ${ }^{1}$, P. Beckerle ${ }^{1}$, C. Bormann ${ }^{1}$, E. Schwab ${ }^{1}$, Y. Shin ${ }^{1}$, R. Stock ${ }^{1}$, H. Ströbele ${ }^{1}$, P. Baltes ${ }^{2}$, \\ C. Müntz ${ }^{2 \star}$, H. Oeschler ${ }^{2}$, C. Sturm ${ }^{2}$, A. Wagner ${ }^{2}$, R. Barth ${ }^{3}$, M. Ciélak ${ }^{3}$, M. Degbowski ${ }^{3}$, E. Grosse $^{3}$, \\ P. Koczoń ${ }^{3}$, M. Mang ${ }^{3}$, D. Miśkowiec ${ }^{3 \star \star}$, R. Schicker ${ }^{3}$, P. Senger ${ }^{3}$, B. Kohlmeyer ${ }^{4}$, F. Pühlhofer ${ }^{4}$, \\ J. Speer ${ }^{4}, K$. Völkel ${ }^{4}$, and W. Walus ${ }^{5}$ \\ 1 Johann Wolfgang Goethe-Universität, D-60325 Frankfurt/Main, Germany \\ 2 Technische Hochschule Darmstadt, D-64289 Darmstadt, Germany \\ 3 Gesellschaft für Schwerionenforschung, D-64220 Darmstadt, Germany \\ 4 Philipps-Universität, D-35037 Marburg, Germany \\ s Jagiellonian University, PL-30-059 Kraków, Poland
}

December 22, 1995

\begin{abstract}
Midrapidity protons from ${ }^{109} \mathrm{Bi}+{ }^{209} \mathrm{Bi}$ collisions were measured with the Kaon Spectrometer at SIS at incident energies of $E_{L a b} / A=400.700$ and $1000 \mathrm{MeV}$. Additionally, light fragments were analysed at $400 \mathrm{MeV}$. We have investigated the azimuthal emission pattern of the particles relative to the reaction plane as function of transverse momentum, bombarding energy and impact parameter. We observe an enhanced emission of particles perpendicular to the reaction plane at all bombarding energies. The ratio of the number of particles emitted out-of-plane/in-plane increases strongly with the particles transverse momentum. The anisotropy decreases with increasing beam energy. Composite particles show a much stronger effect than protons
\end{abstract}

PACS: $25.75 .+r$

\section{Introduction}

Collective flow effects are - besides particle production - the most important tool to investigate hot and compressed nuclear matter produced in relativistic nucleusnucleus collisions $[1,2,3]$. Pioneering experiments have established two kinds of collective effects from the overlap region between two colliding nuclei: The directed flow of nucleons in the reaction plane ("side splash") and the preferred emission of nucleons perpendicular to the reaction plane ("squeeze-out")[4-10]. In particular, the latter is a very promising tool to extract information about the dynamics of hot and compressed nuclear matter. A collective out-of-plane emission was predicted by early hydrodynamical calculations and interpreted as a dynami. cal squeeze out of matter due to the build-up of pressure in the interaction zone of the two colliding nuclei [11] Further theoretical studies in the framework of microscopic models (QMD) found a strong sensitivity to the nuclear equation of state (EOS) for this effect [12].

According to microscopic models [13] many of the observables in heavy-ion reactions are sensitive to both: the EOS and momentum-dependent interactions (MDI).

* Present address: BNL, Upton, NY 11973, USA

* Present address: SUNY, Stony Brook, NY 11794-3800, USA
However, in contrast to flow effects in the reaction plane, the particle flow out-of-plane is expected to be less sensitive to moment um-dependent interactions [14].

Previous experimental results are in qualitative agreement with model calculations. However, quantitative comparisons are difficult since these data are subject to acceptance cuts as well as basic experimental uncertainties in determining the reaction geometry. Therefore, we have analysed the strength of the out-of-plane emission of particles corrected for the uncertainty in determining the reaction plane as a function of transverse momentum. We expect that quantitative comparisons of model calculations with our experimental data will lead to an improved determination of the EOS.

\subsection{Experimental Setup}

The experiment was performed with the Kaon Spectrometer at the Darmstadt (GSI) heavy ion synchrotron SIS [15]. This spectrometer is a double-focussing QD configuration with large solid angle (15-30 msr) and wide momentum acceptance. Particle identification is obtained by measuring momentum and velocity. The momentum is determined from the focal-plane position of the particle trajectories, whereas the velocity is calculated by using information on time-of-flight. Two scintillator detector arrays added to the spectrometer provide additional information to characterize the event [15-18]. The Large Angle Hodoscope gives information on the charged particle multiplicity which is a measure of the impact parameter. This detector consists of 96 modules and accepts charged particles in the polar angular range $12^{\circ}<\theta_{\text {Lab }}<48^{\circ}$. Particles emitted into this angular range are predominantly participating protons. The second external detector, the Small Angle Hodoscope, is located 7 meters downstream of the target. This ar ray is composed of 380 modules and covers polar angles $0.5^{\circ}<\Theta_{L a b}<7^{\circ}$. Most of the particles emitted in this angular range are projectile spectator nucleons. This detector system provides information on polar angle, azimuth, charge and time-of-flight. Projectile spectators are identified by the $\Delta \mathrm{E}$ signal in the scintillator modules and by time-of-flight. The aximuth of the iden- 
tified spectators is used to reconstruct the event plane. We used ${ }^{209} \mathrm{Bi}$ projectiles of $\mathrm{E}_{\mathrm{Lab}} / \mathrm{A}=400,700$ and $1000 \mathrm{MeV}$ with a beam intensity of $10^{5}$ particles per spill and Bi-targets with a thickness of $0.92 \mathrm{~g} / \mathrm{cm}^{2}$ and $0.35 \mathrm{~g} / \mathrm{cm}^{2}$. Figure 1 shows the energy-loss in one of the scintillator paddles of the time-of-flight detector in the focal-plane versus the mass $/ \mathrm{Z}$-ratio of particles detected in the spectrometer. The measurements were done at different magnetic field settings corresponding to the different momentum ranges. The polar angle of the spectrometer was varied to select particle rapidities in the range of $0.35<y / y_{\text {proj }}<0.65$

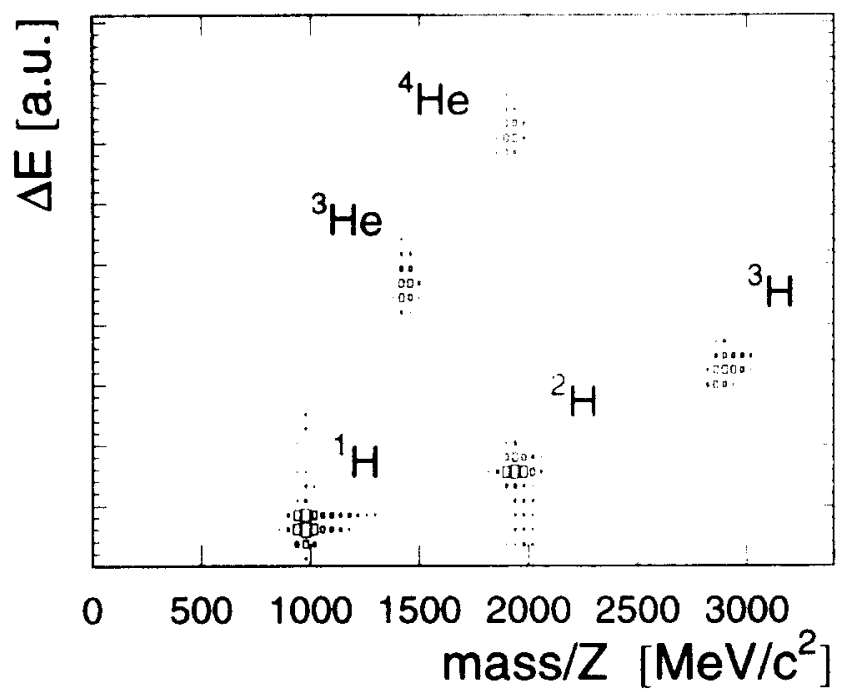

Fig. 1. Energy-loss in one scintillator paddle of the time-of-Alight detector versus the mass/Z-ratio of particles in the spectrometer

\subsection{Impact Parameter Determination}

Following the method devised previously [7] we have divided the multiplicity distribution measured with the Large Angle Hodoscope into five groups (MUL1 to MUL5) to classify the reaction centrality. MUL1(MUL5) indicates the lowest(highest) multiplicity bin corresponding to peripheral(central) collisions. The measured hit multiplicity of charged particles for each multiplicity bin is given in Tab. 1

\begin{tabular}{|c|c|c|c|c|c|}
\hline $\mathrm{E}_{\text {Lab }} / \boldsymbol{A}$ & MUL1 & MUL2 & MUL3 & MUL4 & MUL5 \\
\hline $400 \mathrm{MeV}$ & $<15$ & $15-28$ & $29-41$ & $42-55$ & $>55$ \\
$700 \mathrm{MeV}$ & $<16$ & $16-31$ & $32-47$ & $\mathbf{4 8}-62$ & $>62$ \\
$1000 \mathrm{MeV}$ & $<17$ & $17-33$ & $34-49$ & $50-65$ & $>65$ \\
\hline
\end{tabular}

Table 1. Hit multiplicity range of charged particles measured with the Large Angle Hodoscope in each multiplicity bin used for the impact parameter selection. Note that no multiple hit correction has been applied

Assuming the clean-cut geometrical model [19] the number of participants is a monotonously decreasing function of the impact parameter $b$. The Large Angle Hodoscope covers a wide range of the participant's phase space and therefore the number of participants can be deduced from the measured hit multiplicity of this detector.
For each multiplicity range, MUL1 to MUL5, the number of participating protons $\mathrm{Z}_{\text {part }}$ and the corresponding impact parameters $\langle b\rangle$ are extracted from a simulation which includes the detector geometry of the Large Angle

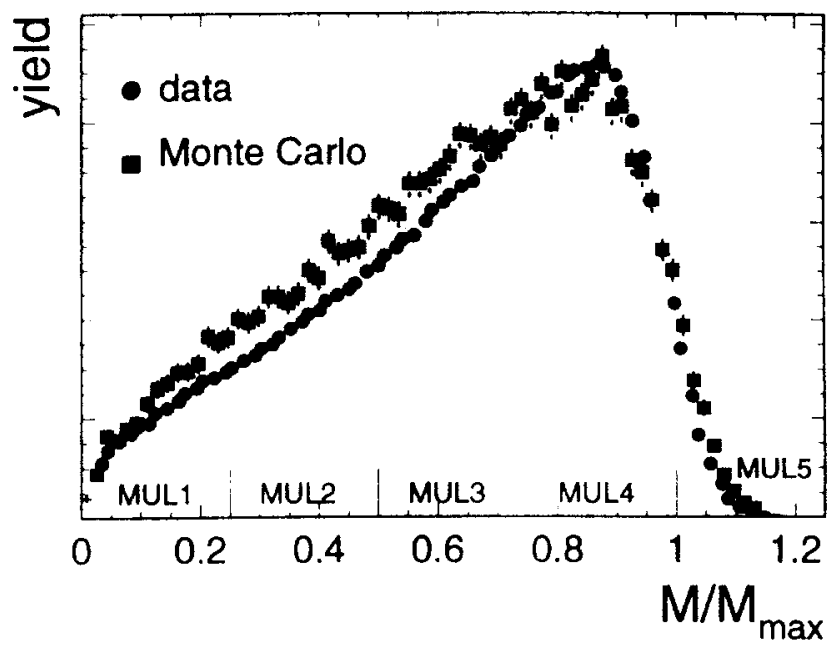

Fig. 2. Vormalized multiplicity distribution of charged particles as measured with the Large Angle Hodoscope at $E_{L a b} / A=400$ $\left(\mathrm{M}_{\max }=55\right)$ and the corresponding values as determined by the simulation. The ordinate is linear starting at zero. Note that the shape of the distribution is biased by the spectrometer trigger.

Hodoscope. An event generator based on the clean-cut model yields a number of charged participants $Z_{\text {part }}$ and pions, respectively, for a given impact parameter $b$. The participating nucleons emitted as $Z=1$ and $Z=2$ particles are assumed to have a Maxwell-Boltzmann momentum distribution in the center of mass system. The particle ratios $\mathrm{He} / \mathrm{H}$ are extracted from the spectrometer data [20]. The momenta are boosted into the laboratory frame according to the incident energy. The calculated

\begin{tabular}{|c|c|c|c|c|c|}
\hline$E_{\text {Lab }} / A$ & MUL1 & MUL2 & MUL3 & MUL4 & MUL5 \\
\hline $400 \mathrm{MeV}$ & $10.9 \mathrm{fm}$ & $8.7 \mathrm{fm}$ & $6.2 \mathrm{fm}$ & $3.4 \mathrm{fm}$ & $1.6 \mathrm{fm}$ \\
$700 \mathrm{MeV}$ & $10.8 \mathrm{fm}$ & $8.6 \mathrm{fm}$ & $6.3 \mathrm{fm}$ & $3.6 \mathrm{fm}$ & $1.6 \mathrm{fm}$ \\
$1000 \mathrm{MeV}$ & $11.0 \mathrm{fm}$ & $9.0 \mathrm{fm}$ & $6.7 \mathrm{fm}$ & $3.9 \mathrm{fm}$ & $1.7 \mathrm{fm}$ \\
\hline
\end{tabular}

Table 2. The average impact parameters $\langle b\rangle$ estimated from the measured hit multiplicity of charged particles in each multiplicity bin. Errors on $\langle b\rangle$ are estimated to be smaller than $\pm 0.3 \mathrm{fm}$ (see text).

hit-multiplicity patterns in the Large Angle Hodoscope reproduce very well the shape of the measured ones. This is shown in Fig. 2 for $\mathrm{E}_{L_{a b}} / \mathrm{A}=400 \mathrm{MeV}$. However, the measured hit multiplicities at $\mathrm{E}_{\text {Lab }} / \mathrm{A}=700 \mathrm{MeV}$ and $1000 \mathrm{MeV}$ are $20 \%$ and $30 \%$, respectively, higher than the simulated ones. This can be explained by additional hits in the detector caused by photons from neutral pions or secondary reaction products. The measured average hit multiplicities increase with increasing beam energies as shown in Tab. 1. From the simulated multiplicity pattern we extracted the corresponding impact parameter distributions. Table 2 shows the average impact parameter $\langle b\rangle$ of the distributions for the multiplicity ranges MUL1 to MUL5. The extracted widths of 
the distributions are approximately $\sigma \approx 1 \mathrm{fm}$. The systematic errors due to particle ratio uncertainties are estimated to be smaller than $\pm 0.3 \mathrm{fm}$. The values of $\langle b\rangle$ are in good agreement with previous results which were obtained by measuring the charged projectile spectators of ${ }^{197} \mathrm{Au}+{ }^{197} \mathrm{Au}$ collisions at $\mathrm{E}_{\text {L, ab }} / \mathrm{A}=1000 \mathrm{MeV}[21]$

\subsection{Reaction Plane Determination}

For the reconstruction of the event plane the transverse momentum method is used [22]. This method yields a reaction plane for each event defined by the beam axis and a vector $\boldsymbol{Q}$, which is the vector sum of the transverse momenta of all spectator particles observed in the Small Angle Hodoscope $[17,21]$. The event plane is characterized by the azimuth $\Phi$. The experimental uncertainty of

\begin{tabular}{|c|r|r|r|}
\hline$E_{\text {Lab }} / \mathrm{A}$ & $400 \mathrm{MeV}$ & $700 \mathrm{Me}$ & $1000 \mathrm{MeV}$ \\
\hline MUL1 & $10.2(5.3)$ & $18.7(8.1)$ & $23.7(10.7)$ \\
MUL2 & $13.9(3.7)$ & $21.2(4.4)$ & $26.3(5.5)$ \\
MUL3 & $11.1(3.4)$ & $16.8(4.2)$ & $218(4.9)$ \\
MUL4 & $5.9(3.0)$ & $96(3.2)$ & $13(1) 4.8)$ \\
MUL5 & $3.7(2.2)$ & $(5.212)$. & $8.8(3.7)$ \\
\hline
\end{tabular}

Table 3. Measured average multiplicity per event of tharged par ticles in the Small Angle Hodoscope used for the reaction plane reconstruction. The values are given for different beam energies and multiplicity ranges. The widths $\tau$ of the corresponding multiplicity distributions are given in brackets.

the event plane reconstruction is estumated by randomly subdividing each event into two subevents, each containing one half of all particles [22]. For each subevent one evaluates the independent vectors $Q_{1}(1=1,2)$ and extracts the angle $\Delta \Phi_{12}=\Phi_{1}-\Phi_{2}$ between the two vectors From the probability distribution $\mathrm{P}\left(\left|\Delta \Phi_{12}\right|\right)$ one can estimate the uncertainty in determining the reaction plane for the complete event.

The procedure is done in two steps. First, the experimental distribution $\mathrm{P}\left(\left|\Delta \Phi_{12}\right|\right)$ is used to estimate the accuracy of the angle $\Delta \Phi_{12}$ between two subevents. In a second step the probability distribution $P\left(\left|\Delta \Phi_{12}\right|\right)$ is reproduced by a Monte Carlo simulation in which the true reaction plane is known and the probability distribution $\mathrm{P}(|\Delta \Phi|)$ between the true and the reconstructed reaction plane is calculated. From this distribution the difference between the true and the reconstructed reaction plane $\Delta \Phi=\Phi_{\text {true }}-\Phi_{\text {est }}$ is estimated. The Monte Carlo procedure takes the detector geometry of the Small Angle Hodoscope into account and simulates a projectile spectator source. The generated event multiplicities as well as the momenta of the particles have been adjusted to reproduce the hit multiplicities (shown in Tab. 3) and transverse momenta of the spectators measured with the Small Angle Hodoscope. The spectator momenta have been generated with a Gaussian momentum distribution. The deflection into the reaction plane is simulated by an additional transverse momentum kick for each particle parallel to the vector $\boldsymbol{Q}$.

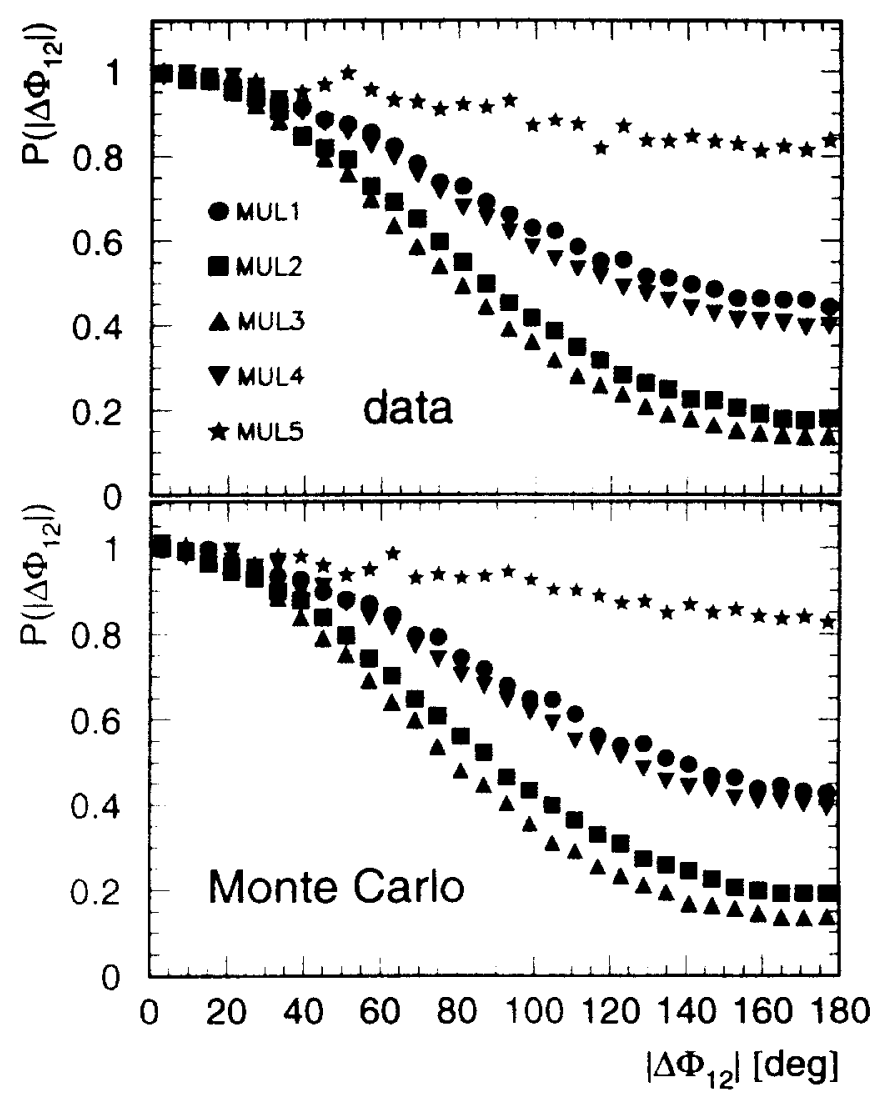

Fig. 3. Probability distribution $P\left(\Delta \Phi_{12}\right)$ for the angular difference $\Delta \Phi_{12}$ between $Q_{1}$ and $Q_{2}$ as measured at a beam energy of $\mathrm{E}_{L a b} / \mathrm{A}=1000 \mathrm{MeV}$ (upper) and simulated (lower). The distributions are shown for different multiplicity ranges.

\begin{tabular}{|c|c|c|c|}
\hline \multicolumn{4}{|c|}{$E_{L a b} / A=400 \mathrm{MeV}$} \\
\hline & $\left\langle\Delta \Phi_{12}^{2}\right\rangle^{1 / 2}$ & $<\Delta \Phi^{2}>^{1 / 2}$ & $\langle\cos (2 \Delta \Phi)\rangle$ \\
\hline MUL1 & $98.2^{\circ}$ & $74.3^{\circ}$ & 0.13 \\
\hline MUL2 & $86.4^{\circ}$ & $51.9^{\circ}$ & 0.35 \\
\hline MLLL3 & $82.1^{\circ}$ & $47.4^{\circ}$ & 0.41 \\
\hline MLL4 & $93.5^{\circ}$ & $67.4^{\circ}$ & 0.18 \\
\hline MLL5 & $100.5^{\circ}$ & $86.5^{\circ}$ & 0.03 \\
\hline \multicolumn{4}{|c|}{$\mathrm{E}_{\text {Lab }} / \mathrm{A}=700 \mathrm{MeV}$} \\
\hline & $\left\langle\Delta \Phi_{12}^{2}\right\rangle^{1 / 2}$ & $<\Delta \Phi^{2}>^{1 / 2}$ & $\langle\cos (2 \Delta \Phi)\rangle$ \\
\hline MULI & $91.7^{\circ}$ & $59.5^{\circ}$ & 0.27 \\
\hline MLL2 & $76.7^{\circ}$ & $40.2^{\circ}$ & 0.49 \\
\hline MLL3 & $75.0^{\circ}$ & $39.7^{\circ}$ & 0.51 \\
\hline MUL4 & $91.7^{\circ}$ & $61.0^{\circ}$ & 0.25 \\
\hline MUL5 & $101.2^{\circ}$ & $85.8^{\circ}$ & 0.04 \\
\hline \multicolumn{4}{|c|}{$\overline{\mathrm{E}}_{\text {Lab }} / \mathrm{A}=1000 \mathrm{MeV}$} \\
\hline & $\left\langle\Delta \Phi_{12}^{2}\right\rangle^{1 / 2}$ & $<\Delta \Phi^{2}>^{1 / 2}$ & $\langle\cos (2 \Delta \Phi)\rangle$ \\
\hline MUL1 & $91.2^{\circ}$ & $54.9^{\circ}$ & 0.30 \\
\hline MUL2 & $77.5^{\circ}$ & $40.0^{\circ}$ & 0.50 \\
\hline MUL3 & $73.1^{\circ}$ & $36.4^{\circ}$ & 0.55 \\
\hline MUL4 & $89.0^{\circ}$ & $55.9^{\circ}$ & 0.31 \\
\hline MUL5 & $101.1^{\circ}$ & $80.5^{\circ}$ & 0.08 \\
\hline
\end{tabular}

Table 4. Uncertainties of the azimuthal difference between the reaction plane calculated from the subevents and between the estimated and the true reaction plane, respectively, as extracted from the simulation (see text). The statistical errors are negligible. 
The upper picture in Fig. 3 shows the measured probability distribution $\mathrm{P}\left(\left|\Delta \Phi_{12}\right|\right)$ between $\boldsymbol{Q}_{1}$ and $\boldsymbol{Q}_{2}$ extracted from the data at a beam energy of $E_{L a b} / A=1000 \mathrm{MeV}$ The lower picture shows the corresponding distribution obtained by the Monte Carlo simulation. The results of the Monte Carlo simulation, given in Tab. 4, show the dispersions $\left\langle\Delta \Phi_{12}^{2}>^{1 / 2}\right.$ and $\left\langle\Delta \Phi^{2}>^{1 / 2}\right.$ of the azimuthal difference $\Delta \Phi_{12}$ and $\Delta \Phi$, respectively. If there is no correlation between the two vectors the values for $\left.<\Delta \Phi_{12}^{2}\right\rangle^{1 / 2}$ and $\left\langle\Delta \Phi^{2}\right\rangle^{1 / 2}$ are about $104^{\circ}$ degrees. The values of $\left\langle\Delta \Phi^{2}\right\rangle^{1 / 2}$ are lower than the values of $<\Delta \Phi_{12}^{2}>^{1 / 2}$. This improvement in accuracy arises from a doubling of the multiplicity and the change from a deviation between two found vectors to a deviation from one found vector $\boldsymbol{Q}[22]$.

\section{Results}

We first demonstrate the azimuthal asymmetry of the observed emission pattern. Figure 4 shows the azimuthal distribution of the vector $\boldsymbol{Q}$ around the beam axis for events for which $\mathrm{H}$-isotopes are observed in the spectrometer. The angle $\varphi$ is the relative azimuthal angle between the estimated reaction plane and the emitted particle. The distributions show maxima around \pm 9()$^{\circ}$ with respect to the event plane. This pattern demonstrates the preferred emission of particles out of the reaction plane. The distributions are parametrized by the expression $N(\varphi) \sim 1+P_{1} \cos (\varphi)+P_{2} \cos (2 \varphi)$.

The parameter $P_{1}$ is related to the in-plane emission of the particles and is zero for particles emitted around midrapidity. The spectrometer acceptance for different particles is not symmetric with respect to midrapidity. In our case the measured protons and deuterons stem predominantly from the forward hemisphere whereas most tritons have backwards rapidities. This results in values of $P_{1}$ different from zero $\left({ }^{1} \mathrm{H}: P_{1}=0.04,{ }^{2} \mathrm{H}: P_{1}=0.03,{ }^{3} \mathrm{H}\right.$ $P_{1}=-0.27$ ).

Negative values of $P_{2}$ indicate an out-of-plane emission. In Tab. 5 the parameters $P_{2}$ for protons are given for the multiplicity bins MUL1 to MLI.4 and different transverse-moment um ranges

The values $P_{2}$ are affected by the uncertainty in determining the reaction plane. The true values $P_{2}^{\prime}$ are larger than the measured $P_{2}$ Therefore. the $P_{2}$ coefficients have been corrected for the fluctuations $\lrcorner \Phi$ of the estimated reaction plane with respect to the true reaction p'ane by the relation [5]. $P_{2}^{\prime}=P_{2} /\langle\cos (2 \Delta \phi)\rangle$. The values $\langle\cos (2 \Delta \Phi)\rangle$ (see Tab. 4) are obtained from the Monte Carlo simulation described in chapter 2.3 . The simulation was tuned until the relevant experimental distributions (cf. Fig. 3) were matched which resulted in vanishing systematical errors of the correction factors (last column of Tab. 4). The corrected values $P_{2}^{\prime}$ are used to quantify the ratio $R_{N}^{\text {corr }}$ of the number of particles emitted perpendicular to the number of particles emitted in the reaction plane: $R_{N}^{c o r r}=\left\{N\left(90^{\circ}\right)+\right.$ $\left.N\left(-90^{\circ}\right)\right\} /\left\{N\left(0^{\circ}\right)+N\left(180^{\circ}\right)\right\}=\left\{1-P_{2}^{\prime}\right\} /\left\{1+P_{2}^{\prime}\right\} . \mathrm{A}$ ratio $R_{N}^{\text {corr }}$ larger than unity implies a preferred outof-plane emission. Figure 5 shows the ratio $R_{N}^{\text {corr }}$ for

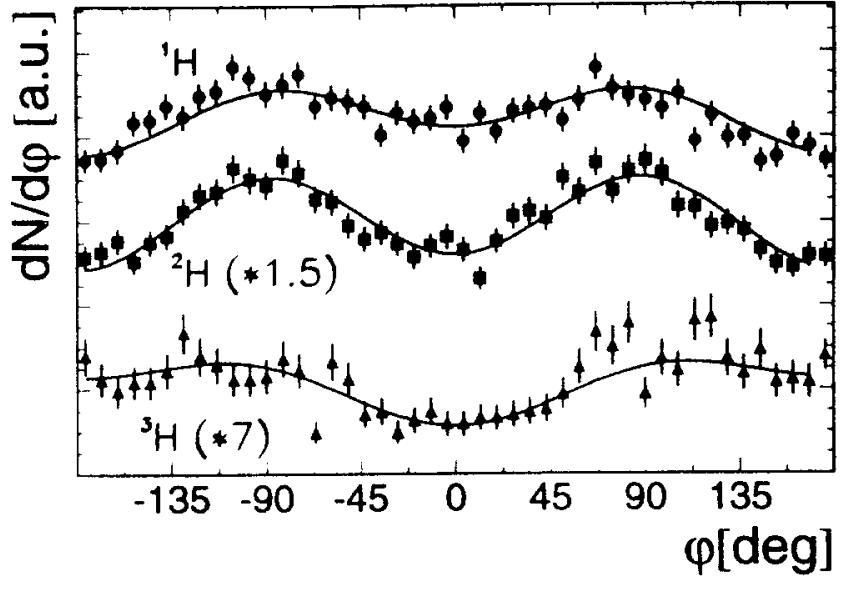

Fig. 4. Typical d $\mathrm{N} / \mathrm{d} \varphi$-distributions for $\mathrm{H}$-isotopes measured at $E_{L_{a b}} / A=400 \mathrm{MeV}$ for semi-central collisions (MUL2,MUL3). The ordinate is linear starting at zero. The particles analysed stem from a momentum range of $400<\mathrm{p}_{T} / \mathrm{A}<500 \mathrm{MeV} / \mathrm{c}$. The solid lines are fits with $\cos (\varphi)$ and $\cos (2 \varphi)$ terms.

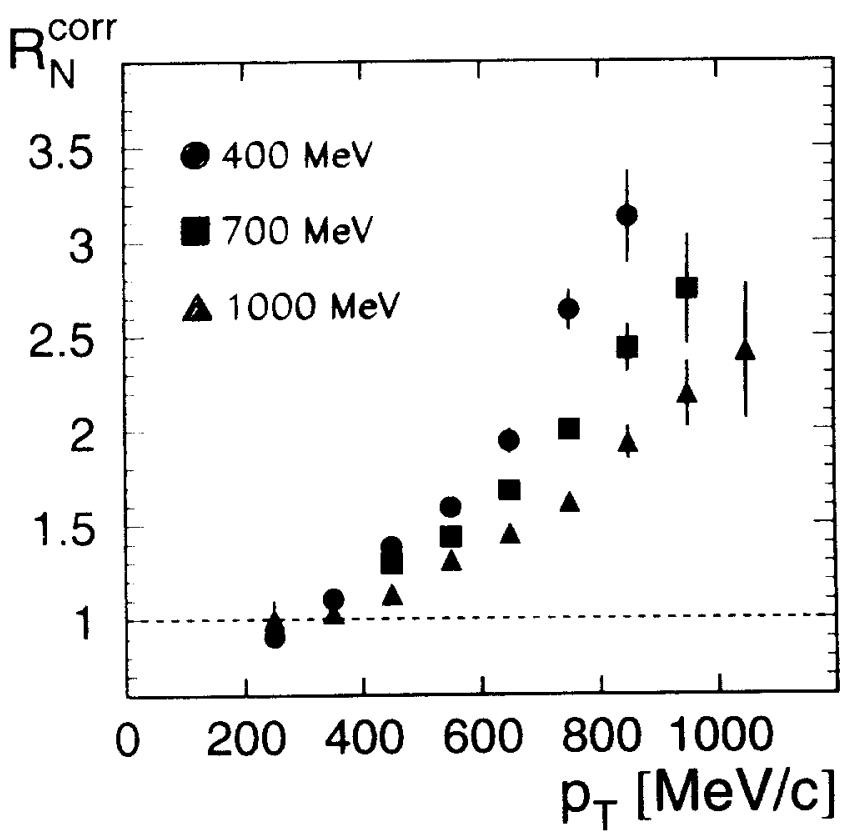

Fig. 5. The corrected out-of-plane/in-plane ratio $R_{N}^{\text {corr }}$ for protons measured at $E_{L_{a b}} / A=400,700$ and $1000 \mathrm{MeV} . \mathrm{R}_{N}^{c o r r}$ is shown as a function of the transverse momentum for semi-central collisions (MUL2,MUL3). The dashed line at $\mathrm{R}_{N}^{\text {corr }}=1$ indicates an isotropic emission at midrapidity

protons as a function of the transverse momentum for different beam energies. The centrality cut corresponds to the sum of the multiplicity bins MUL2 and MUL3. The average impact parameter is estimated to be about $<\mathrm{b}>\approx 7.5 \mathrm{fm}$. A strong dependence of $R_{N}^{\text {corr }}$ on the transverse momentum $p_{T}$ is observed. The ratio increases with increasing $p_{T}$ but decreases with increasing beam energy. The impact parameter dependence of $R_{N}^{c o r r}$ is shown in Fig. 6 for three incident energies. The data indicate a strong dependence on the collision central- 
ity. The strongest anisotropy is observed at the most peripheral collisions (MUL1). This observation is valid for $E_{L a b} / A=700$ and $1000 \mathrm{MeV}$. In the case of MUL1 at $400 \mathrm{MeV}$ the large reaction plane uncertainties (see Tab. 4) would cause relatively large errors on $R_{N}^{\text {corr }}$. Therefore, only the corresponding uncorrected parameter $P_{2}$ is given (Tab. 5).

\begin{tabular}{|c|c|c|c|c|}
\hline \multicolumn{5}{|c|}{$\mathrm{E}_{L a b} / \mathrm{A}=400 \mathrm{MeV}$} \\
\hline$p_{T}[\mathrm{GeV} / \mathrm{c}]$ & MUL1 & MUL2 & MUL3 & MUL4 \\
\hline $0.2-0.3$ & $-0.04(1)$ & $+0.01(1)$ & $+0.03(1)$ & $+0.04(1)$ \\
\hline $0.3-0.4$ & $-0.03(1)$ & $-0.05(1)$ & $+0.00(1)$ & $-0.01(1)$ \\
\hline $0.4-0.5$ & $-0.03(1)$ & $-0.07(1)$ & $-0.06(1)$ & $0.00(1)$ \\
\hline $0.5-0.6$ & $-0.07(1)$ & $-0.12(1)$ & $00 \pi(1)$ & $-0.02(1)$ \\
\hline $0.6-0.7$ & $-0.11(2)$ & $-0.15(1)$ & $.0 .11(1)$ & $-0.03(1)$ \\
\hline $0.7-0.8$ & $-0.09(2)$ & $-0.21(1)$ & $016(1)$ & $-0.04(1)$ \\
\hline $0.8-0.9$ & $-0.14(4)$ & $-0.26(2)$ & $018(3)$ & $-0.04(1)$ \\
\hline $0.9-1.0$ & $-0.17(9)$ & $-0.35(4)$ & $-0.22(5)$ & $-0.06(2)$ \\
\hline $1.0-1.1$ & $-0.04(19)$ & $-0.38(8)$ & $-0.22(11)$ & $-0.08(3)$ \\
\hline \multicolumn{5}{|c|}{$E_{L a b} / A=700 \mathrm{MeV}$} \\
\hline$p_{T}[\mathrm{GeV} / \mathrm{c}]$ & MUL1 & MUL2 & MUL3 & MUL4 \\
\hline $0.2 \cdot 0.3$ & $+0.04(15)$ & $+0.21(13)$ & $+0.01(12)$ & $+0.04(10)$ \\
\hline $0.3-0.4$ & $-0.06(2)$ & $-0.06(1)$ & $-005(1)$ & $+0.01(1)$ \\
\hline $0.4-0.5$ & $-0.08(1)$ & $-0.0911)$ & -1) 0.411 & $0.00(1)$ \\
\hline $0.5-0.6$ & $-0.12(1)$ & -0.13111 & .1) 050111 & $0.00(1)$ \\
\hline $0.6-0.7$ & $-0.15(1)$ & -0.17111 & 150941 & $0.00(1)$ \\
\hline $0.7-0.8$ & $-0.18(2)$ & -0.21111 & .1113111 & $-0.01(1)$ \\
\hline $0.8-0.9$ & $-0.21(3)$ & .0 .29121 & 111501 & $0.04(1)$ \\
\hline $0.9-1.0$ & $-0.27(6)$ & $-0.30(4)$ & $-1) 19(2)$ & $0.03(2)$ \\
\hline $1.0-1.1$ & $-0.22(12)$ & $-0.24(7)$ & $-(3.29(4)$ & $-0.03(3)$ \\
\hline \multicolumn{5}{|c|}{$E_{L a b} / \mathrm{A}=1000 \mathrm{MeV}$} \\
\hline$p_{T}[\mathrm{GeV} / \mathrm{c}]$ & MUL1 & MUL2 & MUL3 & MUL4 \\
\hline $0.2-0.3$ & $-0.07(5)$ & $-0.02(4)$ & $+0.01(3)$ & $+0.04(3)$ \\
\hline $0.3-0.4$ & $-0.05(1)$ & $-0.03(1)$ & $+0.01(1)$ & $+0.04(1)$ \\
\hline $0.4-0.5$ & $-0.08(1)$ & $-0.05(1)$ & $-001(1)$ & $+0.02(1)$ \\
\hline $0.5-0.6$ & $-0.10(1)$ & $-0.10(1)$ & $-0.0411)$ & $+0.02(1)$ \\
\hline $0.6-0.7$ & $-0.12(1)$ & $-0.1411)$ & $-0.07(1)$ & $+0.01(1)$ \\
\hline $0.7-0.8$ & $-0.15(2)$ & $-0.17(1)$ & $0.0911)$ & $0.00(1)$ \\
\hline $0.8-0.9$ & $-0.18(3)$ & $-0.22(2)$ & (i) 13111 & $-0.03(1)$ \\
\hline $0.9-1.0$ & $-0.22(6)$ & $-0.25(3)$ & .0 .15121 & $0.00(2)$ \\
\hline $1.0-1.1$ & $-0.23(11)$ & $-0.27(6)$ & $.0 .18(4)$ & $-0.02(2)$ \\
\hline
\end{tabular}

Table 5. Parameters $P_{2}$ not corrected for the reaction plane uncertainty for events in which protons were observed in the spectrometer in different $p_{T}$-intervals. The statistical errors are given in brackets. The values of MUL5 are comparable with zero within the error bars and therefore not shown

At $\mathrm{E}_{\mathrm{Lab}} / \mathrm{A}=400 \mathrm{MeV}$ not only protons hut also composite particles have been measured. Tahle 6 shows the parameters $P_{2}$ for $\mathrm{H}$ - and He-isotopes for semi-central collisions $(\langle b\rangle \approx 7.5 \mathrm{fm})$. The correction factor for this centrality cut (MUL2, MUL3) is $\langle\cos (2 \Delta \phi)\rangle=0.39$. A comparison of the $\mathrm{H}$-isotopes shows a clear increase of the anisotropy with increasing mass of the fragment. Tritons and ${ }^{3} \mathrm{He}$ show the same anisotropy within the errors.
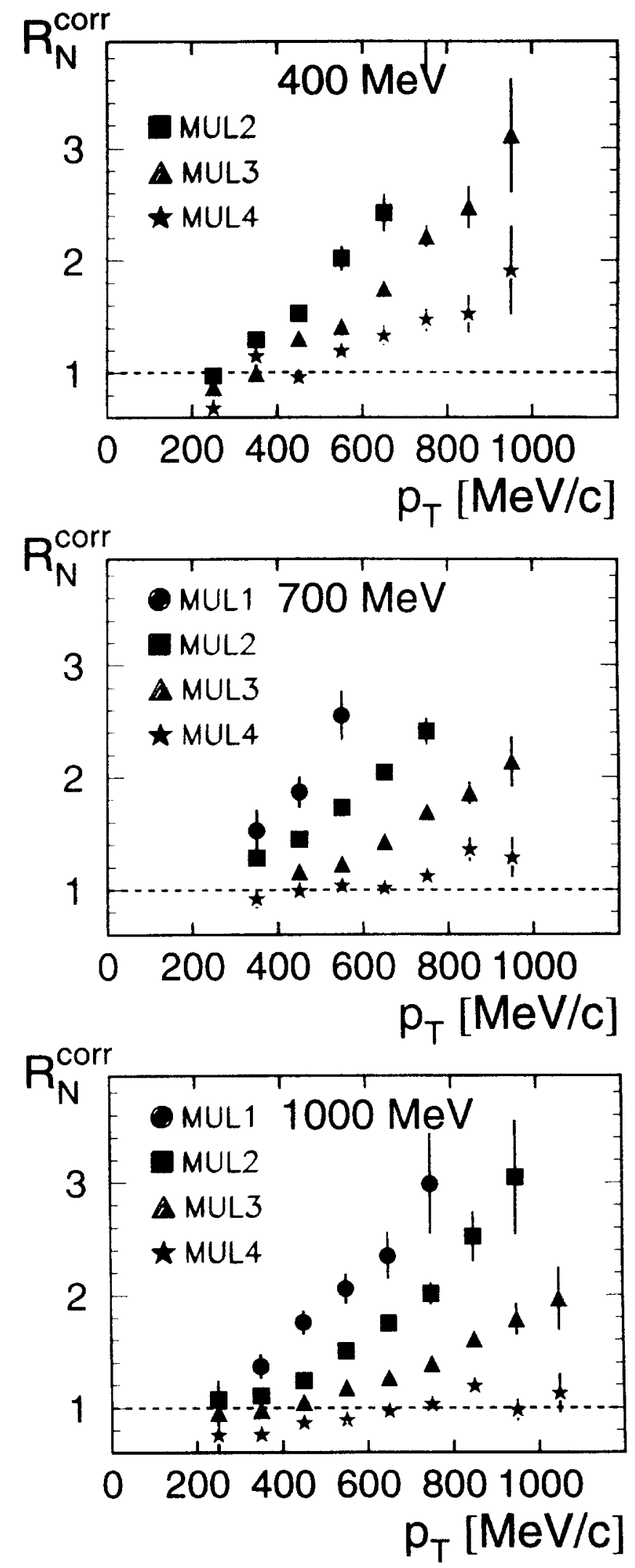

Fig. 6. The out-of-plane/in-plane ratio $R_{N}^{\text {corr }}$ for protons measured at different incident energies $\mathrm{E}_{\text {lab }} / \mathrm{A}$. The $R_{N}^{\text {corr }}$ is shown as a function of the transverse momentum for different multiplicity ranges. The values $R_{N}^{\text {corr }}$ are corrected for the uncertainty in determining the reaction plane. 


\begin{tabular}{|c|c|c|c|c|c|}
\hline \multicolumn{7}{|c|}{$\mathrm{E}_{\text {Lab }} / \mathrm{A}=400 \mathrm{MeV}$} \\
\hline$p_{T} / \mathrm{A}$ & ${ }^{\mathrm{T}} \mathrm{H}$ & ${ }^{2} \mathrm{H}$ & ${ }^{3} \mathrm{H}$ & ${ }^{3} \mathrm{He}$ & ${ }^{1} \mathrm{He}$ \\
\hline $\mathbf{0 . 1 - 0 . 2}$ & - & $\mathbf{0 . 0 4 ( 2 )}$ & $+0.01(3)$ & - & $-0.03(4)$ \\
$\mathbf{0 . 2}-0.3$ & $+0.02(1)$ & $-0.01(1)$ & $-0.06(1)$ & $-0.07(2)$ & $-0.13(1)$ \\
$\mathbf{0 . 3 - 0 . 4}$ & $-0.02(1)$ & $-0.08(1)$ & $-0.15(1)$ & $-0.13(1)$ & $-0.16(1)$ \\
$\mathbf{0 . 4 - 0 . 5}$ & $-0.06(1)$ & $-0.15(1)$ & $-0.22(4)$ & $-0.24(1)$ & $-0.25(2)$ \\
$\mathbf{0 . 5 - 0 . 6}$ & $-0.09(1)$ & $-0.21(1)$ & & $-0.29(3)$ & $-0.34(6)$ \\
$\mathbf{0 . 6 - 0 . 7}$ & $-0.12(1)$ & $-0.27(3)$ & - & $-0.33(7)$ & $-0.58(19)$ \\
$\mathbf{0 . 7 - 0 . 8}$ & $-0.18(1)$ & - & & & - \\
$\mathbf{0 . 8 - 0 . 9}$ & $-0.20(1)$ & - & & & \\
$0.9-1.0$ & $-0.25(2)$ & - & & & \\
$\mathbf{1 . 0 - 1 . 1}$ & $-0.26(4)$ & - & & & \\
\hline
\end{tabular}

Table 6. Parameters $P_{2}$ not corrected for the reaction plane uncertainty for different intervals $\mathrm{p}_{T} / \mathrm{A}[\mathrm{GeV} / \mathrm{c}]$. The values are given for semi-central collisions (MUL2, MUL3). The statistical errors are given in brackets.

\subsection{Discussion}

The energy dependence of the preferred emission out of plane is consistent with previous experiments. The Plastic-Ball collaboration found a maximum signal $R y$ for protons at a beam energy of $E_{L_{a b}} / \mathrm{A}=400 \mathrm{MeV}$ and a decrease at higher incident energies [ 7 ]. Similar findings were reported by the LAND-collaboration for neutrons [9]

The impact parameter dependence shown in Fig. 6 is seemingly in disagreement to the Plastic-Ball data which show a maximum for semi-central collisions [ 7$]$. Our data indicate the strongest effect at the multiplicity bin $M \mathrm{LL} \mathrm{L}$ that corresponds to peripheral collisions. However. the Plastic-Ball data have not been corrected for uncertainties in determining the reaction plane. This reduces the values $R_{N}$ for peripheral collisions since the uncertainties are larger than for semi-central collisions

Moreover, the Plastic-Ball data are shown in the frinctpal axis system of the tri-axial momentum ellipsoid. Due to sidewards flow into the reaction plane the orient ation of this ellipsoid differs by the flow angle $\Theta_{\text {flou }}$ from the orientation of the beam axis. In peripheral collisions the flow is small but increases for decreasing impact parameter [23]. Large flow angles will lead to a lowermg of $\mathbf{R}_{N}$ and $\mathrm{R}_{N}^{\text {corr }}$. respectively, if evaluated relatiw w the beam axis. To get a quantitative understanding of such effects we have performed Monte Carlo simulations in a very simplified schematic model. The results of these are sketched in Fig. 7 in which an emission pattern is simulated that leads to similar ratios as measured at $E_{\text {Lab }} / \mathrm{A}=700 \mathrm{MeV}$. The emission pattern is approx!mated by an ellipsoid in momentum space, whose shape and orientation is defined by the flow angle $\Theta_{\text {fiur }}$ and two aspect ratios $r_{31}$ and $r_{21}$ [24]. The ellipses shown in Fig. 7 are contours of a Gaussian momentum distribution in the center-of-mass system. The axis $r_{2}$ of the ellipsoid coincides with the $y$-axis of the cm-coordinate system, whereas the axes $r_{1}$ and $r_{3}$ are rotated by $\Theta_{\text {flow }}$ versus the $\mathrm{x}$ - and $\mathrm{z}$-axis, respectively. The reaction plane is denoted $\mathrm{p}_{x} \mathrm{p}_{z}$. It is obvious that the in-plane momentum component $p_{x}$ is strongly affected by $\Theta_{\text {flow }}$, whereas the out-of-plane component $p_{y}$ does not change. Figure 8 shows the corresponding out-of-plane/in-plane ratios
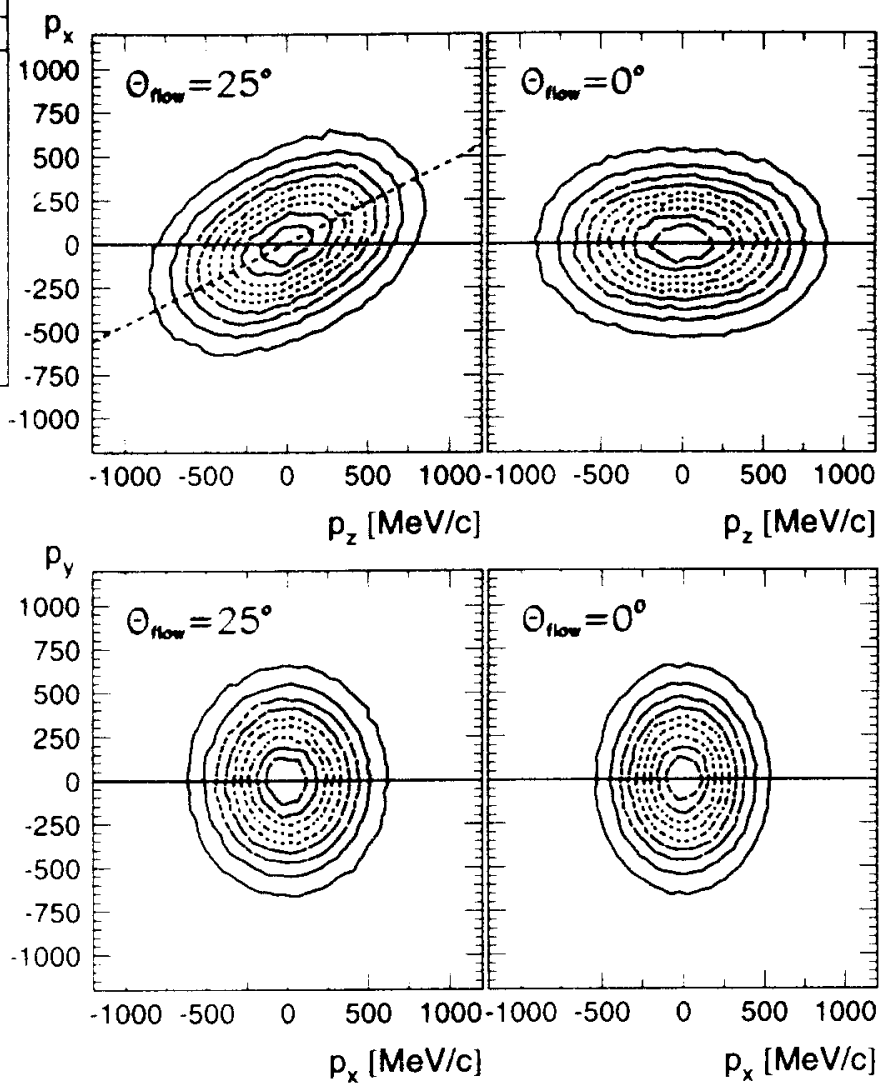

Fig. 7. Contours representing an anisotropic two-dimensional Gaussian momentum distribution obtained from a Monte Carlo simulation. Plotted are the in-plane aspect ratio $r_{31}=1.6$ (upper) and out-of-plane $r_{21}=1.3$ (lower), respectively. On the right, the flow axis (dashed line) is rotated by the flow angle $\Theta_{\text {flow. }}$. The $p_{z}$-axis indicates the beam axis.

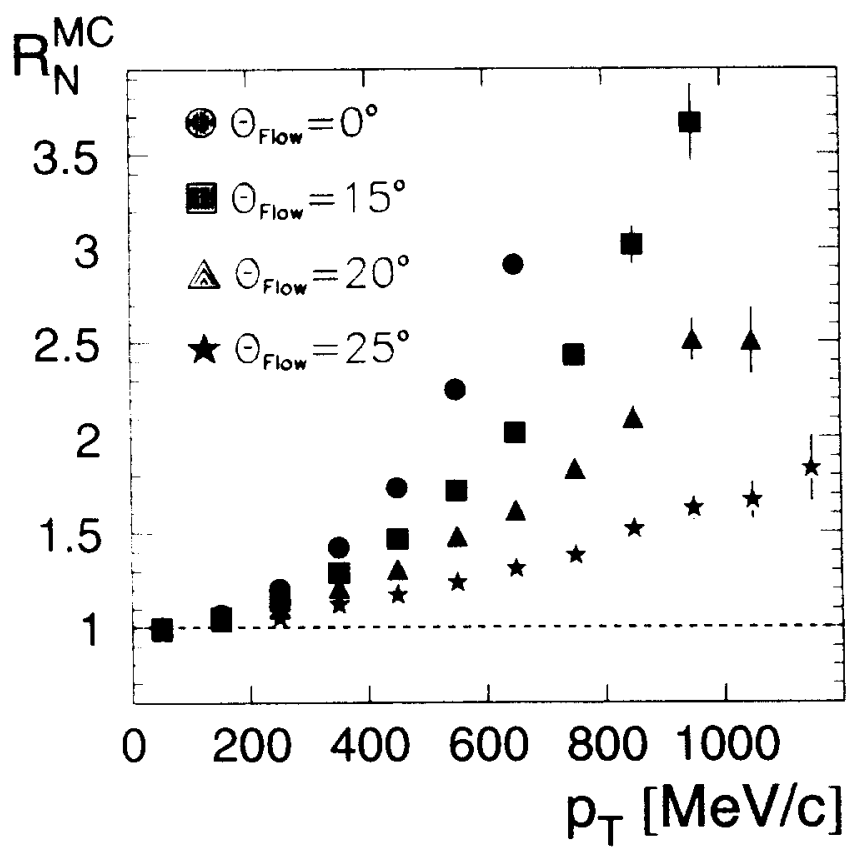

Fig. 8. Simulated out-of-plane/in-plane ratios $\mathrm{R}_{N}^{M C}$ with respect to the beam axis as function of $p_{T}$ for different $\Theta_{\text {flow }}$. 
as a function of transverse momentum for different angles $\theta_{\text {flow }}$ of the momentum ellipsoid with respect to the beam axis. $\Theta_{\text {flow }}=0^{\circ}$ means that the principal axis of the ellipsoid coincides with the beam direction. The figure demonstrates that the ratio $\mathrm{R}_{N}^{M C}$ is reduced in strength if $\Theta_{\text {flow }}$ is increased. The decreasing ratios of the measured data from MUL1 to MUL3 at $E_{L a b} / A=700 \mathrm{MeV}$ (see Fig. 6) can be reproduced by changing the flow angle $\theta_{\text {flow }}$ from 0 to 20 degrees. Therefore, the higher ratios $\mathrm{R}_{N}$ for peripheral (MUL1) compared to semi-central (MUL2, MUL3) collisions might be an artifact caused by higher $\Theta_{\text {flow }}$ in semi-central collisions. Even though the sidewards flow is larger in more central collisions (MUL4), the influence of $\Theta_{\text {flow }}$ on $R_{N}$ is reduced due to a more isotropic configuration of the reaction geometry Small flow angles are associated with elongated shapes $r_{31}>1$, and larger flow angles with shapes closer to spherical symmetry $\left(r_{31} \approx 1\right)[24]$. In the limit of $r_{31} \rightarrow 1$ no influence of $\Theta_{\text {flow }}$ should be observed

These considerations show that for an interpretation of the impact parameter dependence of the ratio $\mathrm{R}_{N}^{\text {corr }}$ around the beam axis the event shape his to be known. For a rotation of the momentum ellipsoid into the prin cipal axis system the flow angle $\Theta_{\text {flou }}$ must be reconstructed on an event-by-event basis This reconstruction can only be done by a sphericity analysis of $4 \pi$ data. Since we observe only one particle pere event in the spectrometer, our data can only be given with respect to the beam axis. It is worth noting that the determmation of the true flow angle is a difficult experimental task even for $4 \pi$ detectors since it is strongly affected by ever present acceptance limitations and the necessarily incomplete separation of spectator nucleons from participants.

In addition the above simulation show's that the in crease of $\mathrm{R}_{N}^{M C}$ with increasing $p_{T}$ can be generated by a phase space distribution based on (iaussian momentum sources assuming different widths for the $p_{x}{ }^{-}$and $p_{y}$ momentum distributions. This means that higher values of $\mathrm{R}_{N}$ at higher transverse momenta can be the trivial consequence of the corresponding phase space distribution. More sophisticated arguments predict that the azimuthal anisotropy vanishes quadratically with momentum as $p_{T}$ tends to zero [25]. Nevertheless. compression effects of nuclear matter might influence the shape of the $p_{T}$-dependence. Indeed, recent studies in the framework of IQMD for neutrons have shown a sensitivity of $R_{x}$ as a function of $p_{T}$ to the EOS not only for data in the principal axis system but also for data taken around the beam axis. Our measured $p_{T}$-dependence is in qualitative agreement with microscopic model predictions [26].

A comparison of the azimuthai asymmetry observed in $\mathrm{H}$-isotopes indicates an approximate linear increase of $P_{2}$ as a function of the fragment mass $A$ (see Tab. 6) This is consistent with previous experiments which found that protons show a less pronounced effect than higher charged particles $[7,10]$. This fact is related to a higher collectivity seen by composite particles, since single nucleons are more affected by thermal motion. ${ }^{3} \mathrm{H}$ and ${ }^{3} \mathrm{He}$ show the same anisotropy within the error bars. This

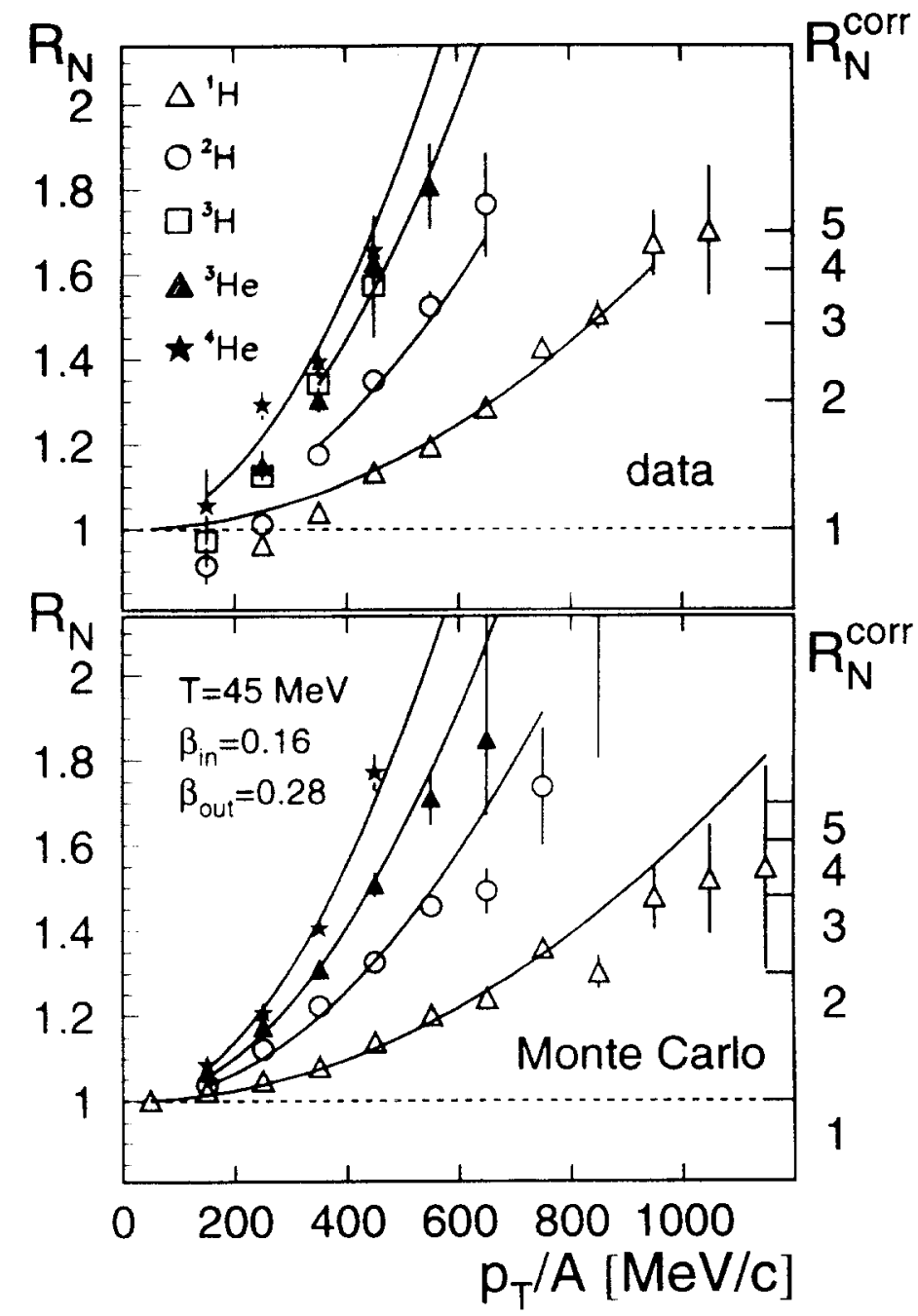

Fig. 9. The upper picture shows the measured out-ofplane/in-plane ratios $R_{N}$ for different particles measured at $\mathrm{E}_{L a b} / \mathrm{A}=400 \mathrm{MeV} . \mathrm{R}_{N}$ is shown as a function of the transverse momentum divided by A for semi-central collisions (MUL2, MUL3). On the right ordinate the values corrected for $s$ the reaction plane $\mathrm{R}_{N}^{c o r r}$ uncertainties are given. The lower picture shows calculated Monte Carlo ratios. The parametrization assumes a source temperature of $T=45 \mathrm{MeV}$ expanding with $\beta_{i n}=0.16$, $\beta_{\text {out }}=0.28$ (lower). The ratios are parametrized by quadratic functions

is clear evidence that Coulomb interactions do not affect the anisotropic emission out of the reaction plane, in accordance to what has been reported for protons and neutrons $[9,10]$.

\subsection{Interpretation by a blast wave scenario}

In the following we will discuss whether the experimental in-plane/out-of-plane ratios are in agreement with a blast wave scenario of nucleons at midrapidity in thermal equilibrium[27]. In this scenario a thermally equilibrated source of protons and composite particles with a temperature $\mathrm{T}$ is superimposed by a blast wave, characterized by a mean radial flow velocity $\beta$. The resulting momentum distribution is given by the expression [28] 


$$
\left.\frac{d^{3} n}{d p^{3}} \sim e^{-\frac{-\gamma}{2}}\left[\frac{\sinh \alpha}{\alpha}\right)(\gamma E+T)-T \cosh \alpha\right]
$$

where $\mathrm{p}$ is the momentum of the particle in the center of mass, $\gamma=\left(1-\beta^{2}\right)^{1 / 2}$, and $\alpha=\gamma \beta p / T$. In contrast to reference [28] we do not consider a spherically symmetric fireball expansion, but an asymmetric expansion with different radial flow velocities in-plane $\beta_{\text {in }}$ and out-ofplane $\beta_{\text {out }}$. The aspect ratios $\mathrm{r}_{21}, \mathrm{r}_{31}$ mentioned in chapter 4.1 can be interpreted as an anisotropic expansion with a higher mean radial flow velocity out-of-plane ( $y$ axis) than in-plane ( $x$-axis) and a higher longitudinal radial flow velocity along the $z$-axis than in-plane, respectively. The variation of the particle momenta resulting in $\mathrm{r}_{21}>1$ leads to a higher particle density $\mathrm{dN} / \mathrm{d} \varphi$ out-of-plane than in-plane. For the interpretation by a blast wave scenario we use the data measured at $400 \mathrm{MeV} / \mathrm{A}$ where we have the data of composite particles with their higher collectivity. At higher bombarding energies composite particles can not be measured at midrapidity because of the limited momentum acceptance of the spectrometer In order to explore the parameters $\mathrm{T}, \boldsymbol{\beta}_{\text {in }}, \boldsymbol{\beta}_{\text {out }}$ describing the data, we compare the results of Monte Carlo calculations with the measured ratios in-plane/out-of-plane $R_{N}$ The parameters $\mathrm{T}, \beta_{\text {in }}, \beta_{\text {out }}$ fulfil the constraint that a fit with a Boltz. mann formula without radial fiow to the particle distribution would yield temperatures around $T_{B o i t z}=65 \mathrm{MeV}$ in accordance to previous experiments[30]. In the blast wave scenario we generated different event samples with source temperatures $\mathrm{T}$ of 40,45 and $50 \mathrm{MeV}$ common for all particle species. The values for $\beta_{n n}$ and $\beta_{\text {out }}$ are chosen such that for the deuterons the simulated ratios agree best to the data. Following $[25,29]$ the calculations are done with a flow angle of $\Theta_{\text {flou }}=20^{\circ}$ The longitudinal radial flow velocity along the $\mathrm{p}_{z}$-axis $\left(\mathrm{r}_{3}\right)$ is assumed to be $\beta_{\|}=0.42$ which is the velocity of the projectile in the center of mass system at the beam energy of $400 \mathrm{MeV} / \mathrm{A}$. This value leads to aspect ratios of approximately $r_{31} \approx$ 1.6. In Fig. 9 the measured (upper) and simulated (lower) ratios are shown. To compare the measurement with the simulation the ratios are parametrized by quadratic functions $R_{N}\left(p_{T}\right) \sim 1+\alpha \cdot p_{T}^{2}[25]$. The resulting fit parameter $a$ as well as the $x^{2}$ per degree of freedom are listed in Table 7 . The best agreement is obtaned by assuming a temperature around $T=45 \mathrm{MeV}$ and radial flow velocities in-plane of $\beta_{\text {in }}=0.16$ and out-of-plane $\beta_{\text {out }}=0.28$ As shown in chapter 4.1 , the extracted values $\beta_{n n}, \beta_{\text {out }}$ depend on $\Theta_{\text {flow }}$ and $\mathbf{r}_{31}$. Deviations from $3_{\mid 1}=0.42$ by $\pm 20 \%\left(r_{31} \approx 1.6 \pm 10 \%\right)$ result in variations of $\pm 15 \%$ in $\beta_{\text {out }}$.

Recent studies done by the EOS-collaboration for $\mathrm{Au}+\mathrm{Au}$ collisions at $\mathrm{E}_{\text {Lab }} / \mathrm{A}=400$. MeV yeld similar results[31]. In these studies temperatures of approximately $\mathrm{T}=55 \mathrm{MeV}$ and a collective radial flow velocity of $\beta_{\text {radial }}=0.22$ were found. The value of $T$ is somewhat higher than our results which can be explained by the fact that the EOS-group has analysed central events. This event class shows an isotropic emission pattern which therefore can be described by one common radial flow velocity $\beta_{\text {radial }}$.

Our calculation suggests that the data can be explained by a scenario assuming a source of protons and composite particles in thermal equilibrium. However, a detailed comparison of the data with the prediction of this schematic model reveals significant differences. The ratio $R_{N}^{c o r r}$ as determined from the experimental data seems to rise only for $p_{T}$ values above $200 \mathrm{MeV} / \mathrm{c}$, whereas the model clearly predicts a rise already at $p_{T}=0$. Apparently the data do not exhibit radial flow in the low momentum particles. The low $p_{T}$-isotropy may be an indication of different dynamics in this part of phase space.

\begin{tabular}{|c|c|c|c|c|c|c|c|c|}
\hline & \multicolumn{2}{|c|}{ data } & \multicolumn{2}{|c|}{$\begin{array}{c}\mathrm{T}=40 \mathrm{MeV} \\
\beta_{i n}=0.19 \\
\beta_{\text {out }}=0.30\end{array}$} & \multicolumn{2}{|c|}{$\begin{array}{c}T=45 \mathrm{MeV} \\
\beta_{1 \mathrm{n}}=0.16 \\
\beta_{\text {out }}=0.28\end{array}$} & \multicolumn{2}{|c|}{$\begin{array}{c}\mathrm{T}=50 \mathrm{MeV} \\
\beta_{\text {in }}=0.11 \\
\beta_{\text {out }}=0.26\end{array}$} \\
\hline id & $x^{2}$ & a & $x^{2}$ & $\mathbf{a}$ & $x^{2}$ & $\mathbf{a}$ & $x^{2}$ & a \\
\hline${ }^{1} \mathrm{H}$ & 3.4 & $0.68(1)$ & 3.3 & $0.66(1)$ & 2.8 & $0.62(1)$ & 0.9 & $0.53(1)$ \\
\hline${ }^{2} \mathrm{H}$ & 2.6 & $1.62(2)$ & 0.8 & $1.63(1)$ & 4.6 & $1.61(2)$ & 3.2 & $1.60(2)$ \\
\hline${ }^{3} \mathrm{H}$ & 2.5 & $2.50(8)$ & 1.0 & $2.39(2)$ & 1.2 & $2.55(2)$ & 3.4 & $2.66(2)$ \\
\hline${ }^{?} \mathrm{He}$ & 1.4 & $2.75(6)$ & - & - & - & - & - & - \\
\hline${ }^{4} \mathrm{He}$ & 1.6 & $3.47(9)$ & 3.9 & $3.11(3)$ & 2.5 & $3.47(3)$ & 1.2 & $3.94(3)$ \\
\hline
\end{tabular}

Table 7. Parameters a of the function shown in Fig. 9. A fit assuming a purely thermal source without radial flow results in a source temperatures of $\mathrm{T}=65 \mathrm{MeV}$ in each case.

\section{Summary}

In summary, we have studied the beam energy and impact parameter dependence of so-called squeeze-out effects for the system ${ }^{209} \mathrm{Bi}+{ }^{209} \mathrm{Bi}$ at projectile energies of $\mathrm{E}_{l a b} / \mathrm{A}=400,700$, and $1000 \mathrm{MeV}$. For the first time particle ratios out-of-plane/in-plane corrected for uncertainties in determining the reaction plane are presented. We observe an enhanced emission of particles perpendicular to the reaction plane. Composite particles exhibit a more pronounced out-of-plane emission: The magnitude of the out-of-plane parameter $\mathrm{P}_{2}$ is proportional to the mass of the particles. Coulomb interactions do not affect the anisotropic emission perpendicular to the reaction plane. The anisotropy is seemingly most pronounced for peripheral collisions and decreases with increasing beam energy. For an interpretation of the magnitude of the out-of-plane/in-plane ratio collective sidewards flow effects have to be taken into account. The experimental data available so far do not allow an unambiguous separation of directed flow and squeeze effects. The increasing $\mathrm{p}_{T}$-dependence of the ratio $\mathrm{R}_{N}$ seems to be the result of decompression expansion effects; it reflects the resulting characteristic phase space distribution of the ejected matter. Finer details of the influence of decompression effects on the $\mathrm{p}_{T}$-dependence of $\mathrm{R}_{N}$ can be extracted only from microscopic model calculations. Their comparison with experimental data is expected to lead to an improved determination of the EOS. For a comparison of the squeeze-out signal shown around the beam axis the models must reproduce the shape of the momentum ellipsoid as well as the sidewards flow correctly. 


\section{Acknowledgement}

The authors are grateful to W'. Reisdorf. (". Hartnack and S. A. Bass for helpful discussions

This work is supported by the German Federal Minister for Research and Technology (BMFT). by the Gesellschaft für Schwerionenforschung (GSI) and by the Polish Committee of Scientific Research

\section{References}

1. R. Stock, Phys. Rep. 135, 259(1986)

2. H. Stöcker and W. Greiner, Phys. Rep 137. 27. (1986)

3. W. Cassing, V. Metag, U. Mosel and K Vitta, Phys. Rep. 188 $365(1986)$

4. H. H. Gutbrod, K. H. Kampert. H. W Kolt, A. M. Poskanzer H. G. Ritter, H. R. Schmidt, Phys Lett 21 BB. $267(1989)$

5. M. Demoulins, D. L'Hote, J. P. Alard, I Augerat, R. Babinet, N. Bastid, F. Brochard, C. Cavata. N. De Marco, P. Dupieux. H. Fanet, Z. Fodor, L. Fraysse, P. Corodetzky. J. Gosset, T. Hayashino, M. C. Lemaire, A. Le Merdy, B. Lucas, J. Mar roncle, G. Montarou, M. J.Parizet, J. Poitou. C Racca W. Schimmerling, Y. Terrien and $O$. Valette. Phys. Lett. B241 476(1990)

6. J. Gosset, M. Demoulins, R. Babinet (Cavata. H. Fanet D. L'Hote, B. Lucas, J. Poitou, M. C Lemaire, J. P. Alard, J. Augerat, N. Bastid, P. Charmensat. P. Dupieux, L. Fraysse. J. Marroncle, G. Montarou, M. J Parizet, D. Qassoud A. Rahmani, F. Brochard, P. Gorodetzky and C. Racca. Phys. Lett. B247, 233(1990).

7. H. H. Gutbrod, K. H. Kampert. B W Kolt, A M Poskanzer H. G. Ritter, R. Schicker, H. H. Schmidt. Phys Rev $\mathrm{C}_{42}$ $640(1990)$.

8. Y. Leifels, T. Blaich, T. W. Elze, H. Emling. H. Freiesleben. K. Grimm, W. Henning, R. Holzmann, J. G. Keller, H. Klingler, J. V. Kratz, R. Kulessa. D. Lambrecht, S. Lange E. Lubkiewicz, E. F. Moore, If Prokopowicz. R Schmidt C. Schütter, H. Spies. K. Stelzer. I. Stroth. E. Wajda W. Walus, M. Zinser, E. Zude, and the F(OPI Collaboration Phys. Rev. Lett. 71, 963(1993)

9. D. Lambrecht, T. Blaich, I It He, H Emling. H. Freiesleben, K. Grimm, W Henning, K. Holzmann. J. G. Keller, H. Klingler, J. V. Kratz. R. Kulessa. S. Lange. Y. Leifels, E. Lubkiewicz. E. F, Nowre. IT Prokopowin. R. Schmidt, C. Schütter, H. spies. K, stelzer. I Stroth, E. Wa jda, W. Walus. M. Zinser, E. Zude, and the Er)PI Collaboration, Z. Phys. A350, 115(1994)

10. A. Kugler, V. Wagner, M. Pachr, M. Sumbera. Yu G. Sobolev. S. Hlavác, R. S. Simon, $R$. Lorencz, $R$. Whigemuth. Phys. Lett. B, 319(1994).

11. H. Stöcker, L. P. Csernai, \& Ciraebnes, i Buchwald, H. Kruse. R. Y. Cusson, J. A Varuhn and W Greiner. Phys. Rev. Lett. 52, 1594(1982)

12. Ch. Hartnack, M. Berenguer, A Jahns, A. V Keitz, R. Mattiello, A. Rosenhauer, J. Schaffner. Th. Schönfeld, H. Sorge. L. Winkelmann, H. Stöcker and W. Greiner, Vucl. Phys. A 538 . 53c(1992).

13. G. Welke, M. Prakash, T. T. S. Kuo, S. Das (iupta and C. Gale, Phys. Rev. C38,2101(1988).

14. C. Hartnack, J. Aichelin, H. Stöcker, W Greiner, Phys. Lett. B336, 131(1994).
15. P. Senger, W. Ahner, P. Baltes, P. Beckerle, C. Bormann, D. Brill, M. Cieślak, E. Grosse, W. Henning, P. Koczoń, B. Kohlmeyer, W. Konrad, D. Miśkowiec, C. Müntz, H. Oeschler, H. Pöppl, W. Prokopowiecz, F. Pühlhofer, S. Sartorius, R. Schicker, B. Schlei, E. Schwab, Y. Shin, J. Speer, J. Stein, K. Stiebing, R. Stock. H. Ströbele, C. Sturm, K. Völkel, A. Wagner, W. Waluś. Vucl. Instr. Meth. A 327, 393(1993).

16. W. Ahner, P. Baltes, C. Bormann, D. Brill, R. Brockmann, 11. Cieślak. E Grosse, W. Henning. P. Koczoń, B. Kohlmeyer, W. Konrad. D. Miśkowiec, C. Müntz. H. Oeschler, H. Pöppl, W. Prokopowiecz, F. Pühlhofer, R. Renfordt, A. Sandoval, $\therefore$ Sartorius, R. Schicker, E. Schwab. P. Senger, Y. Shin, J. Stein, K. Stjebing, R. Stock, H. Ströbele, K. Völkel, A. Wagner, W. Waluś, Z. Phys. A 341, 125(1991).

17 I) Brill, W. Ahner, P. Baltes. R. Barth, C. Bormann, II. Cieślak. M. Dębowski, E. Grosse, W. Henning, P. Koczoń, B. Kohlmeyer, D. Miśkowiec, C. Münız, H. Oeschler, H. Pöppl, F. Pühlhofer, S. Sartorius, R. Schicker, P. Senger, Y. Shin, J. Speer, J. Stein, K. Stiebing, R. Stock, H. Ströbele, K. Völkel, A. Wagner, W. Waluś, Phys. Rev. Lett. 71, 336(1993).

18 [) Miśkowiec, W. Ahner, R. Barth. II ( ieślak, M. Desbowski, E. Grosse, W. Henning, P. Koczon. R. Schicker, E. Schwab, $P$ Senger, P. Baltes, C. Müntz, H. Oeschler, S. Sartorius, Sturm, A. Wagner, P. Beckerle. C. Bormann, D. Brill, $Y$ Shin, J. Stein, R. Stock. H. ströbele, B. Kohlmeyer, 11. Pöppl, F. Pühlhofer, J. Speer, K. Völkel, W. Waluś, Phys Rev. Lett. 72, 3650(1994)

19. J Gosset, H. H. Gutbrod, W. G. Meyer, A. M. Poskanzer, A. Sandoval, R. Stock and G. D. Westfali, Phys. Rev. C16, $629(1977)$.

20. J Speer et al., (to be published).

21. D. Brill, PhD Thesis, Universität Frankfurt/M.(1993), GSI report 93-36 (ISSN 0171-4546)

22. P. Danielewicz and G. Odyniec, Phys. Lett. 157B, 147(1985).

23. H A. Gustafsson, H. H. Gutbrod, B. Kolb, H. Löhner, B. Ludewigt. A. M. Poskanzer, T. Renner, H. Riedesel, 1f G. Ritter, A. Warwick, F, Weik, and H. Wieman, Phys. Rev. Lett. 52, 1590(1984).

24. P. Danielewicz, H. Ströbele, G. Odyniec, D. Bangert, R. Bock, R. Brockmann, J. W. Harris, H. G. Pugh, W. Rauch, K. E. Renfordt, A. Sandoval, D. Schall, L.S. Schroeder, and R. Stock. Phys. Rev. C38, 38(1988)

25. P' Danielewicz, Phys. Rev. C51, T16(1995).

26. $\therefore$ A. Bass, C. Hartnack, H. Stöcker, W. Greiner. \%. Phys A352, 171(1995).

2. J. P. Bondorf. S. I. A. Garpman, and J. Zimanyi, Vucl. Phys. A296, 320(1978)

$28 \mathrm{I} .1$. Siemens and J. O. Rasmussen. Phys. Rev. Lett. 42. $880(1979)$.

29 Proceedings of a NATO Advanced Study Institute on The Nu- lear Equation of State, Peniscola. Spain, NATO ASI Series B: Physics 216B(1989)

30. K. H. Kampert, in Proceedings of the International Workshop on Gross Properties of Nuclei and Nuclear Excitation XVI, Hirschegg, Austria, 1988, edited by H. Feldmeier (GSI, ISSN 0720-8715, Darmstadt, Germany, 1988).

31 M. A. Jisa, S. Albergo, F. Bieser, F. P. Brady, Z. Cac(1a. D). A. Cebra, A. D. Chacon, J. L. Chance, Y. Choi, S. Costa. J. B. Elliott, M. L. Gilkes, J. A. Hauger, A. S. Hirsch, E. L. Hjort, A. Insolia, M.Justice, D. Keane, J. Kintner. H. S. Matis, M. McMahan, C. McParland, D. L. Olson, M. D. Partlan, N. T. Porile, R. Potenza, G. Rai, J. Rasmussen, H. G. Ritter, J. Romanski, J. L. Romero, G. V. Russo, R. Scharenberg, A. Scott, Y. Shao, B. K. Srivastava, T. J. M. Symons, M. Tincknell, C. Tuvé, S. Wang, P. Warren, G. D. Westfall, H. H. Wieman, and K. Wolf (EOScollaboration), Phys. Rev. Lett. 75, 2664(1995). 